\title{
Modeling and Two-Input Sliding Mode Control of Rotary Traveling Wave Ultrasonic Motors
}

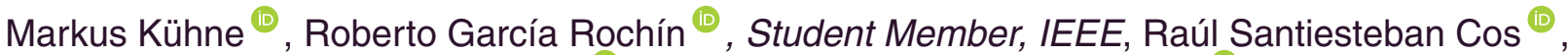 \\ Guillermo Javier Rubio Astorga $^{\mathbb{D}}$, Member, IEEE, and Angelika Peer ${ }^{\mathbb{1}}$, Member, IEEE
}

\begin{abstract}
Traveling wave ultrasonic motors are actuators relying on piezoelectric ceramics that combine many advantageous features, such as high stalling torque, fast response, compactness, and magnetic resonance compatibility. However, they suffer from nonlinear dynamics, loaddependent dead zones, and the difficulty to control low speeds. In this paper, we present a novel second-order model for traveling wave ultrasonic motors. It is based on a dry friction driving principle and features dead zone effects. Based on the model, a two-input sliding mode controller is designed. It controls both phase difference and frequency of the traveling wave, without the necessity of implementing a signum function. With this controller, the state-of-the-art is extended to the position control case, while at the same time using fine-grained phase difference control for low velocities. Moreover, we show global uniform asymptotic stability for bounded disturbances and that velocity jumps do not appear when the control domains of phase difference and frequency are switched. Finally, both the model and the controller are evaluated via simulations and experiments that include the response to a position step input under various opposing torques.
\end{abstract}

Index Terms-Control engineering, piezoelectric resonators, switched systems.

\section{INTRODUCTION}

$\mathbf{T}$ RAVELING wave ultrasonic motors (USMs) are actuators based on high frequency vibration of a stator that is pressed against a rotor. The stator is a deformable body, carrying piezoelectric ceramics that create a traveling wave. The traveling wave is composed of two standing waves with frequency $f$ and phase difference $\alpha$. Force is transmitted to the rotor via a friction interface. USMs combine compactness with a high stalling torque. They also feature a fast response, silent

Manuscript received October 16, 2016; revised February 2, 2017, April 25, 2017, August 3, 2017, and November 6, 2017; accepted January 12 , 2018. Date of publication January 26, 2018; date of current version May 1 , 2018. This work was supported by the German Research Foundation (DFG) within the project "VR-system for visuo-haptic stimulation during fMRI studies." (Corresponding author: Markus Kühne.)

M. Kühne is with the Technical University of Munich, 80333 Munich, Germany (e-mail: Markus.Kuehne@tum.de).

R. García Rochín, R. Santiesteban Cos, and G. J. Rubio Astorga are with the Tecnológico Nacional de México, Culiacán 06080, Mexico (e-mail: rober_rgr@ hotmail.com; raulsncos@gmail.com; grubio@gdl. cinvestav.mx).

A. Peer is with the Free University of Bozen-Bolzano, 39031 Bolzano, Italy (e-mail: Angelika.Peer@brl.ac.uk).

Color versions of one or more of the figures in this paper are available online at http://ieeexplore.ieee.org.

Digital Object Identifier 10.1109/TIE.2018.2798570 operation, and can be exposed to strong magnetic fields, such as in a magnetic resonance (MR) scanner. Hence, they have been employed in robotic, aerospace, and medical applications as well as in camera lenses [1]. Moreover, new structures are being developed [2], [3] that exploit the virtues of this type of actuator and push the possible applications to new limits. Like other researchers [4], [5], we are interested in applying this type of motor in MR environments. However, a series of disadvantages prohibit their application in many cases: USMs have highly nonlinear dynamics, low speeds are difficult to control, dead zones appear when load is added to the rotor, their performance deteriorates at high temperatures [6], and their lifetime is short compared to dc motors.

In this paper, we aim for a model-based controller to employ USMs in an MR-compatible haptic interface. This means that both low and high speeds have to be realized, since the operator should be able to perform both slow and fast movements. Moreover, a varying load is added to the rotor that simulates an opposing torque, resulting from a varying human impedance or virtual environment. Dead zones and nonlinearities are compensated for by a controller that does not require a torque measurement. Torque sensors increase the footprint in the MR environment and the likeliness of image artifacts. Low and high speeds are realized by using the phase difference and frequency as control inputs.

A range of dynamic models of USMs has been proposed, taking into account rotor, stator, and friction dynamics. However, the interactions between the components are often overly complex for control design [1], [7], [8].

Simpler USM models have been proposed for control purposes that abstract the stator dynamics as a torque source and mostly neglect friction as well as dead zone dynamics [6], [9]-[13]. Moreover, Canudas-de-Wit [14] published a model based on work by Hagood and McFarland [7] that attempts to close the gap between model complexity and control development, resulting in a velocity source model. García-Rochín et al. [15] proposed a modification to this model, including a dynamic description of the dead zone and modeling the friction interface via viscous friction, abstracting the stator as a torque source.

Based on the published USM models, a variety of controllers have been developed. Those cover PI-control [16], $\mathrm{H} \infty$ control [17], backstepping control [18], sliding mode control (SMC) [9], [14], [19], control by neural networks [20], and fuzzy logic [21]. The controllers use phase difference, frequency, voltage of the piezo-ceramics, or a mixture of these as control 
variables. Most of the controllers rely on models that neglect the dead zone dynamics and that consider the stator as a torque source [9], [17]-[19]. This simplifies the friction dynamics to a large extent. Hence, the design of the controllers lacks completeness, especially for applications with a varying opposing torque. Canudas-de-Wit [14] proposed a velocity controller based on his velocity source model, where the control variable is mainly the frequency of the traveling wave, while the sign of the phase difference is used to set the direction of rotation. However, only high velocities can be realized with frequency control. Controllers with multiple control inputs as proposed in [9] and [19] on the other hand lack a stability analysis of the resulting hybrid system. Moreover, practical issues like velocity-discontinuities arise for these approaches when the control variable is switched, as will be shown in this paper.

In this paper, we introduce a novel second-order model for traveling wave ultrasonic motors that reproduces dead zone effects and includes a dry friction driving principle. Moreover, a two-input SMC is developed that allows the control of both phase difference and frequency of the traveling wave. It does not require an explicit digital implementation of a signum function, since this function is already part of the motor model. Thus, the controller avoids chattering phenomena that are typical for SMCs. The controller extends the work by Canudasde-Wit [14] to the position control case and allows fine-grained phase difference control. Moreover and unlike previous work, the controller does not introduce velocity jumps when the control domains of phase difference and frequency are switched. The latter is especially desirable for haptic interfaces, where the operator should not feel transitions. Finally, the model is identified with a Shinsei ${ }^{1}$ USR60 and the controller is evaluated experimentally.

This paper is organized as follows. In Section II, USM dynamics is derived. The two-input sliding mode controller is introduced in Section III and a stability analysis is carried out in Section IV. The experimental setup, the parameter identification, and the controller validation are presented in Section V and VI. Finally, a conclusion is drawn in Section VII.

\section{DYNAMIC MODEL}

USMs consist of three major components: Stator, rotor, and friction interface. A free body diagram of the components is depicted in Fig. 1. Here, the stator is subject to a high frequency voltage $V_{0}$ actuating the piezoelectric ceramics and generating a traveling wave. The rotor is pressed on the stator by an axial force $F$. The tangential force that is transmitted to the rotor results in a driving torque $\tau_{d r}$. An opposing torque $\tau_{\text {op }}$ is created when load is added to the rotor.

Canudas-de-Wit [14] described the USM by

$$
\begin{aligned}
J_{r} \ddot{\theta}_{r}+C_{r} \dot{\theta}_{r} & =r_{0}\left(\sigma_{0} \xi+\sigma_{1} \dot{\xi}\right)=\tau_{d r}(\xi, \dot{\xi}) \\
\dot{\xi} & =\omega_{\mathrm{st}}-\dot{\theta}_{r}-\sigma_{0} \frac{\left|\omega_{\mathrm{st}}-\dot{\theta}_{r}\right|}{g\left(\omega_{\mathrm{st}}-\dot{\theta}_{r}\right)} \xi
\end{aligned}
$$

\footnotetext{
${ }^{1}$ [Online]. Available: http://www.shinsei-motor.com
}

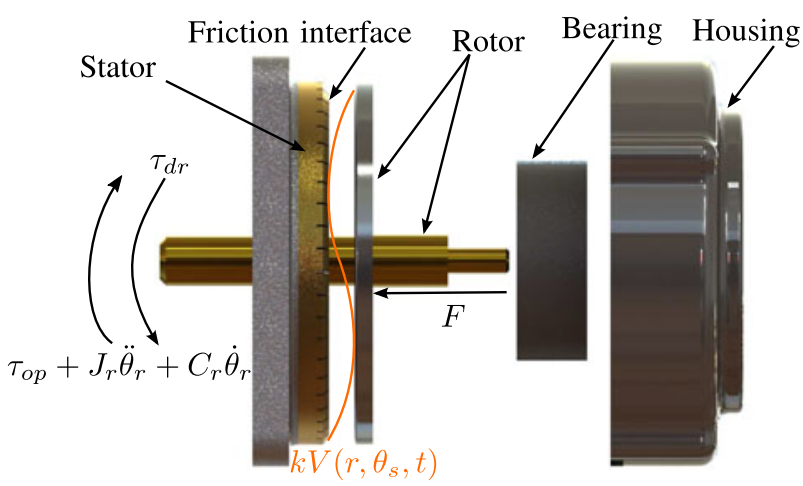

Fig. 1. Free body diagram of a USM, in this case a Shinsei USR60.

$$
\begin{aligned}
g\left(\omega_{\mathrm{st}}-\dot{\theta}_{r}\right) & =F_{C}+\left(F_{S}-F_{C}\right) e^{\frac{-\left(\omega_{\mathrm{st}}-\dot{\theta}_{r}\right)^{2}}{\omega_{0}^{2}}} \\
\omega_{\mathrm{st}} & =V_{0} R\left(f, \omega_{n}\right) \operatorname{sgn}(\alpha) .
\end{aligned}
$$

In this model, (1) describes the rotor dynamics, (2), (3) represent the friction dynamics, and (4) is the stator velocity. The moment of inertia of the rotor $J_{r}$, the friction coefficient $C_{r}$, and the mean radius $r_{0}$ of inner and outer radius of the rotor area of contact are parameters of the rotor dynamics. The friction state $\xi$ constitutes, together with $r_{0}$ as well as the spring-like micro-damping friction coefficients $\sigma_{0}$ and $\sigma_{1}$, the driving friction torque $\tau_{d r}$. The LuGre friction model abstracts the friction interface as a contact between bristles and the mean deflection of the bristles is characterized by the friction state $\xi$. The model relies on several variables and parameters: The rotor velocity $\dot{\theta}_{r}$, the stator velocity $\omega_{\text {st }}$, the Stribeck velocity $\omega_{0}$, the Coulomb friction $F_{C}$, and the Stribeck friction $F_{S}$. The stator is modeled as a velocity source and its dynamics is simplified. Hence, the stator velocity $\omega_{\text {st }}$ is a function of the voltage applied to the piezoelectric material $V_{0}$, as well as the frequency of the traveling wave $f$, and the resonant frequency of the piezoelectric material $\omega_{n}$. It also depends on the sign of the phase difference $\alpha$. The nonlinear dependence on the frequency of the traveling wave and the resonant frequency of the piezoelectric material is described by $R\left(f, \omega_{n}\right)$. This model allows controlling the motor using the frequency of the traveling wave, the voltage applied on the piezoelectric material, and the sign of the phase difference. It does not include load or the possibility to control the phase difference in the whole range of $\left[-\frac{\pi}{2} ; \frac{\pi}{2}\right]$ rad. More details on the model by Canudas-de-Wit [14] can be found in Appendix A. In this paper, this model is first simplified to adjust the complexity for control design. Then, it is extended to include the effects of load and a varying phase difference.

\section{A. Simplification of the Dynamic Model by Canudas-de-Wit}

The LuGre friction model [22] characterizes a series of known friction properties, such as Stribeck effect, stick-slip motion, and produces an approximated hysteresis curve. However, it comes with an elevation of the system's order and makes control design difficult. In this paper, we aim at establishing a model using a simpler friction dynamics that still reproduces the major 
features of a USM and that can be used for control design. According to [14] the friction dynamics is much faster than the rotor dynamics. Hence, it can be assumed that

$$
\begin{gathered}
\dot{\xi}=0 \\
\xi=\frac{1}{\sigma_{0}} g\left(\omega_{\mathrm{st}}-\dot{\theta}_{r}\right) \operatorname{sgn}\left(\omega_{\mathrm{st}}-\dot{\theta}_{r}\right) \\
\operatorname{sgn}(x)= \begin{cases}1, & x>0 \\
{[-1,1],} & x=0 \\
-1, & x<0\end{cases}
\end{gathered}
$$

using Orlov's [23] definition of the signum function. With these assumptions (1) is reduced to

$$
J_{r} \ddot{\theta}_{r}+C_{r} \dot{\theta}_{r}=r_{0} g\left(\omega_{\mathrm{st}}-\dot{\theta}_{r}\right) \operatorname{sgn}\left(\omega_{\mathrm{st}}-\dot{\theta}_{r}\right) .
$$

Further assuming that $F_{C}=F_{S}$ and substituting $\tau_{m}=r_{0} F_{C}$, the simplified model can be written as

$$
J_{r} \ddot{\theta}_{r}+C_{r} \dot{\theta}_{r}=-\tau_{m} \operatorname{sgn}\left(\dot{\theta}_{r}-\omega_{\mathrm{st}}\right)=\tau_{d r} .
$$

This model describes the friction dynamics as pure dry friction and its solutions are understood in the Filippov sense [24].

\section{B. Extension of the Dynamic Model by Canudas-de-Wit}

The original model by Canudas-de-Wit does not include a load torque that leads to dead zone effects. Since we would like to design a controller for a haptic interface, where variable opposing torques will be created by the human and the rendered virtual environment, it is necessary to include this effect in the model. Therefore, the rotor dynamics (9) is extended by an opposing torque $\tau_{\mathrm{op}}$. Thus, the extended rotor dynamics is expressed as

$$
J_{r} \ddot{\theta}_{r}+C_{r} \dot{\theta}_{r}=-\tau_{\mathrm{op}}+\tau_{d r}=-\tau_{\mathrm{op}}-\tau_{m} \operatorname{sgn}\left(\dot{\theta}_{r}-\omega_{\mathrm{st}}\right)
$$

with $\omega_{\text {st }}$ as control input. The stator velocity $\omega_{\text {st }}$ can be modulated by phase difference changes in the whole range of $\left[-\frac{\pi}{2} ; \frac{\pi}{2}\right]$ rad and it is assumed that the dependency can be modeled by a sinusoidal function [15], [25]. The dependency on the frequency is further assumed to be exponential [15] with the two parameters $a$ and $b$. Parameter $a$ is the maximum frequency of the traveling wave, whereas parameter $b$ describes how rapidly the stator velocity changes when the frequency is altered. Hence, the ideal stator velocity is described by

$$
\omega_{i}=\sin (\alpha) e^{a-b f} .
$$

However, dead zones caused by an opposing torque occur in the phase difference domain [15] of the stator velocity $\omega_{\mathrm{st}}$ as depicted in Fig. 2. Here, these dead zones are modeled analogously to [25], [26]. The stator velocity is therefore expressed as

$$
\omega_{\mathrm{st}}= \begin{cases}z\left(\tau_{\mathrm{op}}\right)\left[\sin (\alpha)-\sin \left(\alpha_{\mathrm{dz}}\left(\tau_{\mathrm{op}}\right)\right)\right] \ldots & \\ \ldots\left[e^{a-b f}-\left|\sin \left(\alpha_{\mathrm{dz}}\left(\tau_{\mathrm{op}}\right)\right)\right|\right], & |\alpha|>\left|\alpha_{\mathrm{dz}}\left(\tau_{\mathrm{op}}\right)\right| \\ 0, & |\alpha| \leq\left|\alpha_{\mathrm{dz}}\left(\tau_{\mathrm{op}}\right)\right|\end{cases}
$$

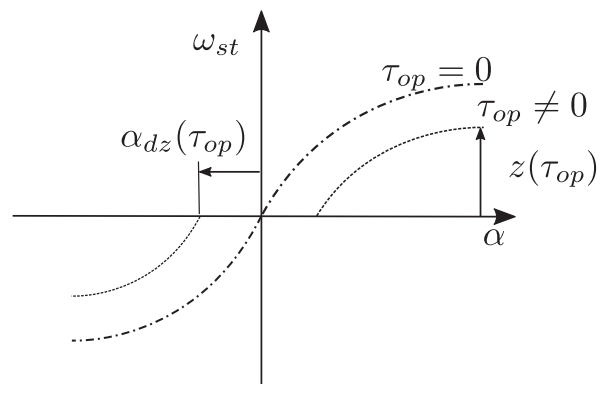

Fig. 2. Dead zone caused by an opposing torque in the phase difference domain.

which describes the nonlinear impact of the opposing torque $\tau_{\mathrm{op}}$ on the stator velocity $\omega_{\mathrm{st}}$. The opposing torque $\tau_{\mathrm{op}}$ blocks the stator which results in a dead zone of width $\alpha_{\mathrm{dz}}\left(\tau_{\mathrm{op}}\right)$. Moreover, the reduced maximum velocity under load is accounted for by $z\left(\tau_{\mathrm{op}}\right)$. For simplicity, (12) can be expressed as

$$
\omega_{\mathrm{st}}=\omega_{i}+\delta\left(f, \alpha, \tau_{\mathrm{op}}\right) .
$$

\section{Two-InPUt SLIDING Mode CONTROLler}

Based on the second-order USM model (10)-(12), a two-input sliding mode controller has been designed. It controls both phase difference and frequency to achieve low and high velocities and does not require the implementation of a signum function. The controller extends the work by Canudas-de-Wit [14] to the position control case and allows fine-grained phase difference control. Moreover, we show its global uniform asymptotic stability (GUAS) for bounded disturbances and unlike other controllers it does not introduce velocity jumps when the control domains of phase difference and frequency are switched. The latter is especially desirable for haptic interfaces, where the operator should not feel transitions. In order to study the output tracking problem of the USM, (10)-(12) is expressed in its state space form

$$
\begin{aligned}
\dot{x}_{1} & =x_{2} \\
\dot{x}_{2} & =-\frac{1}{J_{r}}\left(\tau_{m} \operatorname{sgn}\left(\dot{\theta}_{r}-\omega_{\mathrm{st}}\right)+C_{r} \dot{\theta}_{r}\right)+p\left(\dot{\theta}_{r}, \tau_{\mathrm{op}}, t\right) \\
y & =x_{1} .
\end{aligned}
$$

Here, $\omega_{\mathrm{st}}$ is the control input, $x_{1}=\theta_{r}-r_{\text {ref }}$ is the position error, $x_{2}$ the velocity error, and $p\left(\dot{\theta}_{r}, \tau_{\mathrm{op}}, t\right)=-\frac{\tau_{\mathrm{op}}}{J_{r}}-\ddot{r}_{\mathrm{ref}}$ is a perturbation term.

\section{A. Control Laws}

For a first-order sliding mode controller with sliding surface $S$, the control domains of phase difference and frequency control are separated by $\mu=\dot{\theta}_{r}-S$. The magnitude of $\mu$ determines the control domain, while the switching boundary is at $\mu=1$. The control laws are obtained by inverting the exponential and the sinusoidal functions in (11) depending on frequency and phase difference, respectively, and are summarized in Table I. The control laws allow stabilizing (14), (15) and a smooth switching as will be shown later. 
TABLE I

CONTROLS LAWS in the FREQUENCY AND THE PhASE DifFERENCE DOMAIN

\begin{tabular}{lc}
\hline \hline Frequency domain $(|\mu| \geq 1)$ & Phase difference domain $(|\mu|<1)$ \\
\hline$f=\frac{1}{b}(a-\ln (|\mu|))$ & $f=\frac{a}{b}$ \\
$\alpha= \pm \frac{\pi}{2}$ & $\alpha=\arcsin (\mu)$ \\
\hline \hline
\end{tabular}

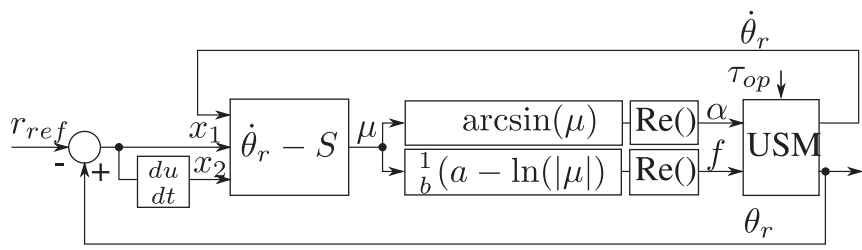

Fig. 3. Control scheme of the two-input SMC.

Substituting the control laws into (14), (15) and with (13), the error dynamics in both the frequency and the phase difference domain becomes

$$
\begin{aligned}
& \dot{x}_{1}=x_{2} \\
& \dot{x}_{2}=-\frac{1}{J_{r}}\left(\tau_{m} \operatorname{sgn}(\tilde{S})+C_{r} \dot{\theta}_{r}\right)+p\left(\dot{\theta}_{r}, \tau, t\right)
\end{aligned}
$$

and the system is forced to follow $\tilde{S}$. Here, $\tilde{S}=S-\delta(f$ $\left.\left(x_{1}, x_{2}\right), \alpha\left(x_{1}, x_{2},\right), \tau_{o p}\right)=S-\delta\left(x_{1}, x_{2}, \tau_{o p}\right)$ and the governing dynamics is determined by $S$. Thus, the control scheme can be depicted as in Fig. 3. Beyond the domain of definition of arcsin and ln, only their real parts are used, leading to the control laws as shown in Table I.

Remark: No signum function is implemented as is usually done for SMCs, since the model of the USM already includes this in its dry friction driving principle. Moreover, no explicit switching is necessary since the controller output $\mu$ naturally switches between the control domains.

\section{B. First Order Sliding Mode}

The error dynamics (17), (18) describes a second-order system with a first-order sliding mode. An ideal linear sliding surface is induced by $S=x_{2}+m x_{1}$, with $m>0$. However, the controlled system will not follow this ideal sliding surface $S$, but deviate by a magnitude of $\delta\left(x_{1}, x_{2}, \tau_{\mathrm{op}}\right)$. Considering (12), this is expressed by a drop of the maximum reachable velocity caused by $z\left(\tau_{\mathrm{op}}\right)$ as well as a parallel shift of a the linear slope in both control domains, caused by $\sin \left(\alpha_{\mathrm{dz}}\left(\tau_{\mathrm{op}}\right)\right)$. Ultimately, a steady-state error remains, which will be quantified later. The sliding surface $\tilde{S}$ is an attractive region and we will show that (17), (18) will slide through its surface as a first-order dynamical system. In order to prove this and thus, that the system is GUAS for bounded disturbances and reaches a set of equilibrium points, the common quadratic Lyapunov function

$$
V(\mathbf{x}, t)=\frac{1}{2} \tilde{S}^{2}
$$

as in [27] and [28] is chosen for both frequency and phase difference domain. Its derivative with respect to time is

$$
\dot{V}(\mathbf{x}, t)=\tilde{S} \dot{\tilde{S}} .
$$

Conditions $V(\mathbf{x}, t)>0$ and $\dot{V}(\mathbf{x}, t)<0$ with $\tilde{S} \neq 0$ have to be fulfilled to show attraction by the sliding surface and stability in the sense of Lyapunov. The first condition is always true since the Lyapunov function is quadratic. In the following sections, it will be shown that the second condition $\dot{V}(\mathbf{x}, t)<0$ is fulfilled for a linear sliding surface.

\section{StABILITY ANALYSIS}

\section{A. Reachability Condition}

With (17), (18), $S=x_{2}+m x_{1}$, and $x_{2}=\dot{\theta}_{r}-\dot{r}_{\text {ref }}$ the reachability condition (20) is then expressed as

$$
\begin{aligned}
\dot{V}=\tilde{S} & \left(-\frac{\tau_{m}}{J_{r}} \operatorname{sgn}(\tilde{S})+\left(m-\frac{C_{r}}{J_{r}}\right) \dot{\theta}_{r}-m \dot{r}_{\mathrm{ref}}\right. \\
& \left.+p\left(\dot{\theta}_{r}, \tau_{\mathrm{op}}, t\right)-\dot{\delta}\left(x_{1}, x_{2}, \tau_{\mathrm{op}}\right)\right) .
\end{aligned}
$$

The perturbation $p\left(\dot{\theta}_{r}, \tau_{\text {op }}, t\right)-\dot{\delta}\left(x_{1}, x_{2}, \tau_{\text {op }}\right)+\left(m-\frac{C_{r}}{J_{r}}\right) \dot{\theta}_{r}-$ $m \dot{r}_{\text {ref }}$ has an upper bound $\mid p\left(\dot{\theta}_{r}, \tau_{\text {op }}, t\right)-\dot{\delta}\left(x_{1}, x_{2}, \tau_{\text {op }}\right)+$ $\left(m-\frac{C_{r}}{J_{r}}\right) \dot{\theta}_{r}-m \dot{r}_{\text {ref }} \mid<M_{l}$. For the special case of $m=\frac{C_{r}}{J_{r}}$ the state dependency vanishes and no estimate of the maximum achievable velocities $\dot{\theta}_{r}$ and the tracking error $x_{2}$ is necessary. In any case, the upper bound of the reachability condition is

$$
\begin{aligned}
\dot{V} & <\tilde{S}\left(-\frac{\tau_{m}}{J_{r}} \operatorname{sgn}(\tilde{S}) \pm M_{l}\right) \\
& =-|\tilde{S}|\left(\frac{\tau_{m}}{J_{r}} \mp M_{l} \operatorname{sgn}(\tilde{S})\right) \\
& <-|\tilde{S}|\left(\frac{\tau_{m}}{J_{r}}-M_{l}\right) .
\end{aligned}
$$

In order to show $\dot{V}(\mathbf{x}, t)<0$, the condition that must be satisfied is

$$
\tau_{m}>J_{r} M_{l} \quad \forall \quad \tilde{S} \neq 0 .
$$

\section{B. Equilibrium Set and Steady-State Error}

From condition (23) an equilibrium set $\Omega$ can be identified for $m x_{1}=\delta\left(x_{1}, x_{2}, \tau_{\mathrm{op}}\right)$ and $x_{2}=0$. It results from the definition of the stator velocity (12) and the resulting dead zone in the phase difference domain as shown in Fig. 2. Thus, the opposing torque leads to a steady-state position error $\Delta x_{1}$ for $x_{2}=0$. The precision of the controller can be estimated by solving (12) using the steady-state condition $\omega_{\text {st }}=\dot{r}_{\text {ref }}=0$. With the control laws in Table I, the stator velocity in the phase difference domain is expressed as

$$
\omega_{\mathrm{st}}=z\left(\tau_{\mathrm{op}}\right)\left[\mu-\sin \left(\alpha_{\mathrm{dz}}\left(\tau_{\mathrm{op}}\right)\right)\right]\left[1-\sin \left(\alpha_{\mathrm{dz}}\left(\tau_{\mathrm{op}}\right)\right)\right] .
$$

Since $\mu$ is defined as

$$
\mu=\dot{\theta}_{r}-S
$$

it can be reformulated with $S=x_{2}+m x_{1}$ to

$$
\mu=\dot{\theta}_{r}-x_{2}-m x_{1} \text {. }
$$


Using $\dot{r}_{\text {ref }}=\dot{\theta}_{r}-x_{2}, \mu$ finally becomes

$$
\mu=\dot{r}_{\text {ref }}-m x_{1} .
$$

With this expression for $\mu$ we can reformulate (24) to

$\omega_{\mathrm{st}}=z\left(\tau_{\mathrm{op}}\right)\left[\dot{r}_{\mathrm{ref}}-m x_{1}-\sin \left(\alpha_{\mathrm{dz}}\left(\tau_{\mathrm{op}}\right)\right)\right]\left[1-\sin \left(\alpha_{m}\left(\tau_{\mathrm{op}}\right)\right)\right]$

In steady state $\omega_{\text {st }}=\dot{r}_{\text {ref }}=0$ and under the condition that $\sin \left(\alpha_{\mathrm{dz}}\left(\tau_{\mathrm{op}}\right)\right)<1$, the steady-state error can be estimated as

$$
\left|\Delta x_{1}\right|=\mid \frac{1}{m}\left(\sin \left(\alpha_{\mathrm{dz}}\left(\tau_{\mathrm{op}}\right)\right) \mid\right. \text {. }
$$

Thus, the equilibrium set is defined as

$$
\Omega=\left\{\mathbf{x} \in \mathbb{R}^{2} \quad|\quad| x_{1}|\leq| \frac{1}{m}\left(\sin \left(\alpha_{\mathrm{dz}}\left(\tau_{\mathrm{op}}\right)\right) \mid, x_{2}=0\right\} .\right.
$$

\section{Smooth Switching of the Variable Structure System}

Sliding modes and velocity jumps should not occur along the switching boundary. This can be ensured by guaranteeing a transversal intersection [29] at the switching boundary. The condition for a transversal intersection is

$$
\mathbf{n}^{T}(\mathbf{x}) \mathbf{f}_{+}(\mathbf{x}) \cdot \mathbf{n}^{T}(\mathbf{x}) \mathbf{f}_{-}(\mathbf{x})>0
$$

where $\mathbf{n}^{T}(\mathbf{x})$ is the normal to the switching boundary and $\mathbf{f}_{ \pm}$is the state vector of the variable structure system on both sides of the switching boundary. From (25) it is known that the condition for switching $\mu$ is only a function of the reference velocity $\dot{r}_{\text {ref }}$ and the position error $x_{1}$. Thus, $\mathbf{n}^{T}(\mathbf{x})=\left[\begin{array}{ll}0 & 1\end{array}\right]^{T}$. Here, three situations in which the switching may occur have to be analyzed for smoothness in the states. This can be a switching during the reaching phase on either side of the phase portrait or a switching during the sliding phase on the sliding surface. Therefore (17) and (18) are written in state space form $\mathbf{f}_{ \pm}$, when $\alpha$ approaches $\frac{\pi}{2} \operatorname{rad}$ and $f$ approaches $\frac{a}{b}$.

1) Case 1. Reaching Phase, $\tilde{S}<0$ : In the first case, the system is in the reaching phase and $\tilde{S}<0$. The dry friction term takes the form of $\operatorname{sgn}(\widetilde{S})=-1$ and the state representation at the switching boundary is

$$
\mathbf{f}_{ \pm}=\left(\begin{array}{c}
x_{2} \\
-\frac{1}{J_{r}}\left(-\tau_{m}+p\left(\dot{\theta}_{r}, \tau_{\mathrm{op}}, t\right)\right)
\end{array}\right) .
$$

2) Case 2. Reaching Phase, $\tilde{S}>0$ : In the second case, the system is in the reaching phase and $\tilde{S}>0$. The dry friction term takes the form of $\operatorname{sgn}(\tilde{S})=1$ and the state representation at the switching boundary is

$$
\mathbf{f}_{ \pm}=\left(\begin{array}{c}
x_{2} \\
-\frac{1}{J_{r}}\left(\tau_{m}+p\left(\dot{\theta}_{r}, \tau_{\mathrm{op}}, t\right)\right)
\end{array}\right) .
$$

3) Case 3. Sliding Phase, $\tilde{S}=0$ : In the third case, the system is on the sliding surface and reduced to a first-order dynamical system. The Filippov continuation method is applied and the simplified dynamics of the system is described by

$$
S=x_{2}+m x_{1}=\delta\left(x_{1}, x_{2}, \tau_{\text {op }}\right)
$$

TABLE II

Characteristic Figures of the Experimental Setup

\begin{tabular}{lc}
\hline \hline Parameter & Value \\
\hline USM model & USR60-E3NT \\
Rated (maximum) speed & $100 \mathrm{rpm}(150 \mathrm{rpm})$ \\
Rated (maximum) torque & $0.5 \mathrm{~N} \cdot \mathrm{m}(1 \mathrm{~N} \cdot \mathrm{m})$ \\
Driving frequency & {$[41 ; 44] \mathrm{kHz}$} \\
Driving phase difference & {$\left[-\frac{\pi}{2} ; \frac{\pi}{2}\right] \mathrm{rad}$} \\
Torque sensor model & $\mathrm{TS} 70$ \\
Rated sensed torque & $2 \mathrm{~N} \cdot \mathrm{m}$ \\
Magnetic brake model & FAS21 \\
Maximum brake torque & $2 \mathrm{~N} \cdot \mathrm{m}$ \\
\hline \hline
\end{tabular}

and then the state representation of the original second-order system takes the form of

$$
\mathbf{f}_{ \pm}=\left(\begin{array}{c}
x_{2} \\
\dot{\delta}\left(x_{1}, x_{2}, \tau_{\mathrm{op}}\right)-m x_{2}
\end{array}\right) .
$$

In all three cases, the gradients of the two controlled systems align at the switching instant, which provides smooth switching without velocity jumps.

\section{EXPERIMENTAL IDENTIFICATION OF the DYNAMIC MODEL}

In order to identify (10)-(12) and to validate the controller, a testbed has been set up as in [15]. It allows the simulation of opposing torques and to control both phase difference and frequency, while recording the velocity of the motor. On the testbed, a USM with attached encoder is connected to a torque sensor and magnetic brake. The USM is a USR60-E3NT from Shinsei. It provides a rated speed of $100 \mathrm{rpm}$ with a maximum of $150 \mathrm{rpm}$. The rated torque is $0.5 \mathrm{~N} \cdot \mathrm{m}$, whereas the maximum torque is $1 \mathrm{~N} \cdot \mathrm{m}$. The motor driver has been modified such that both frequency and phase difference of the traveling wave can be controlled: The phase difference can be set within the range of $\left[-\frac{\pi}{2} ; \frac{\pi}{2}\right] \mathrm{rad}$ and the frequency within the range of $[41 ; 44] \mathrm{kHz}$. The attached encoder can measure 4000 pulses/round in quadrature. The torque sensor is a TS70 from ME-Messsysteme and disposes of a rated torque of $2 \mathrm{~N} \cdot \mathrm{m}$. The magnetic brake of type FAS 21 from LIEDTKE Antriebstechnik can generate a maximum torque of $2 \mathrm{~N} \cdot \mathrm{m}$. The details of the testbed are summarized in Table II. The workstation is equipped with a Mecovis I/O card that generates two voltage bands in the range of $[-10 ; 10] \mathrm{V}$. The motor driver converts these voltage bands to the phase difference and frequency of the traveling wave. The Mecovis I/O card also sets the opposing torque and reads the position signals from the encoder as well as the torque signal from the torque sensor. MATLAB/Simulink is used for command generation and the compiled code is run with a Linux real-time kernel at a sampling rate of $1 \mathrm{kHz}$.

The signal flow between workstation and testbed is depicted in Fig. 4.

The hardware setup of the testbed is depicted in Fig. 5. It shows how motor, encoder, torque sensor, and magnetic brake are connected.

The original and modified motor driver are shown in Fig. 6. An SMD board has been designed for this purpose and it is equipped with some of the original components, but also new 


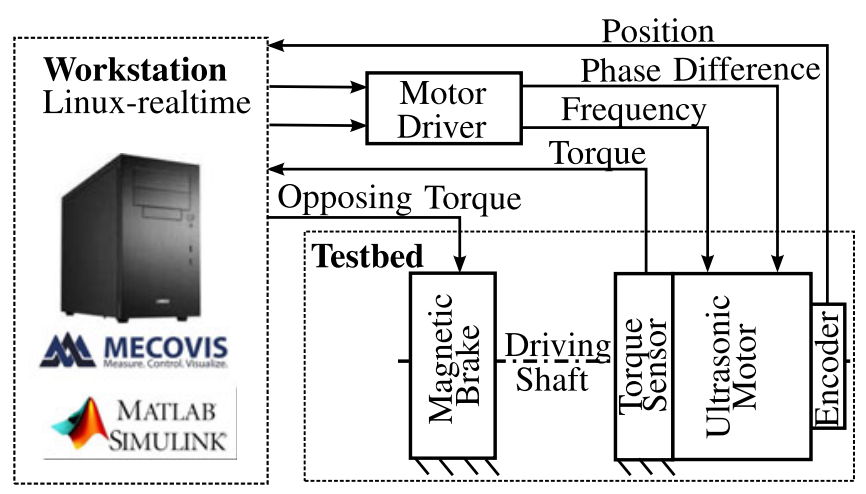

Fig. 4. Scheme of the signal flow between workstation and testbed.

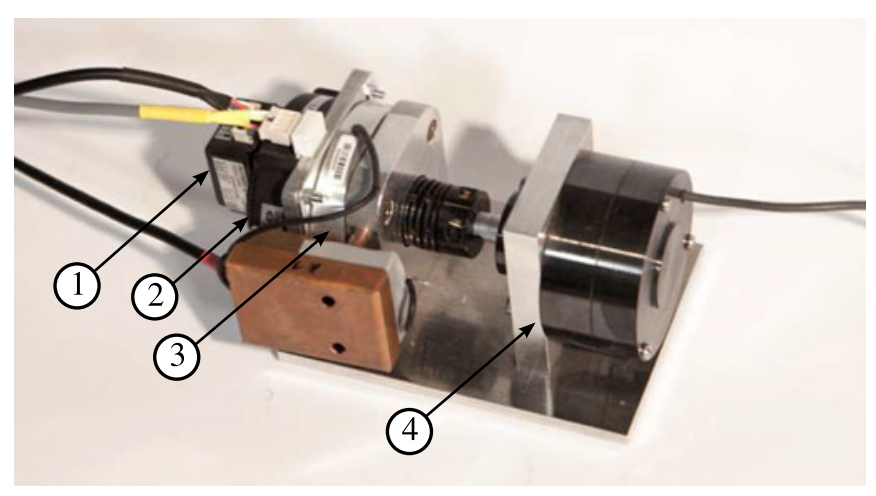

Fig. 5. Photograph of the testbed with (1) Encoder, (2) USM, (3) torque sensor, and (4) magnetic brake.

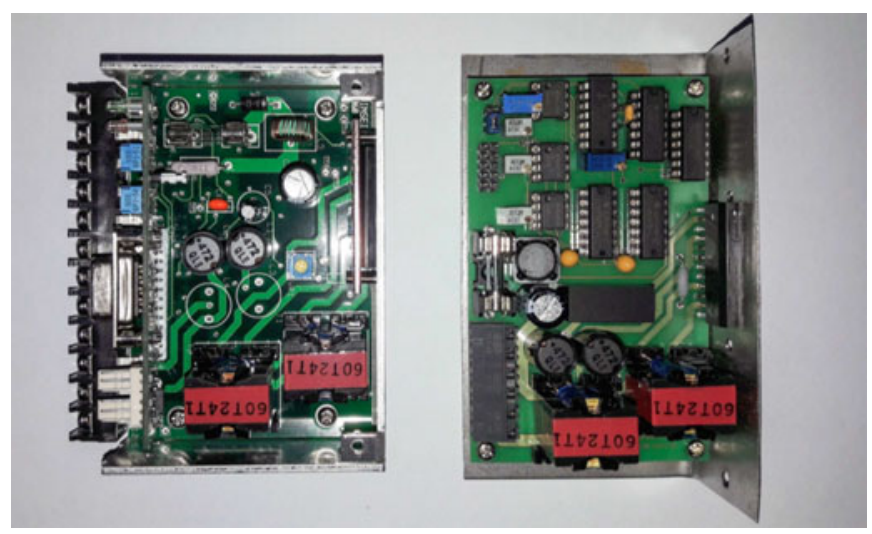

Fig. 6. Photograph of the original (left) and modified (right) motor driver.

ICs to realize fine-grained phase difference control in addition to frequency control.

\section{A. Experimental Procedures}

During the data recording, the phase difference and frequency of the motor are controlled and an opposing torque is generated. A range of curves is recorded: The phase difference is controlled continuously in the range of $\left[-\frac{\pi}{2} ; \frac{\pi}{2}\right] \mathrm{rad}$ and the frequency at steps of $0.5 \mathrm{kHz}$ in the range of $[41 ; 44] \mathrm{kHz}$. The magnetic brake allows us to set a load torque at eleven different levels. This procedure has been adopted from [15] to obtain comparable results and considers only opposing torques below the rated

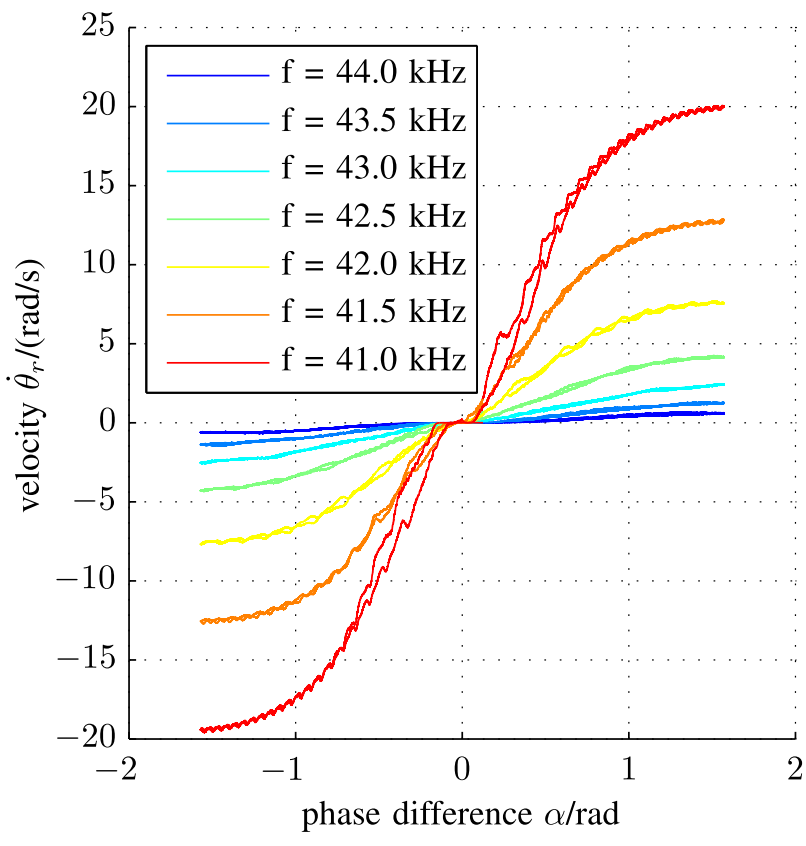

Fig. 7. USM velocity depending on phase difference and frequency at minimum load.

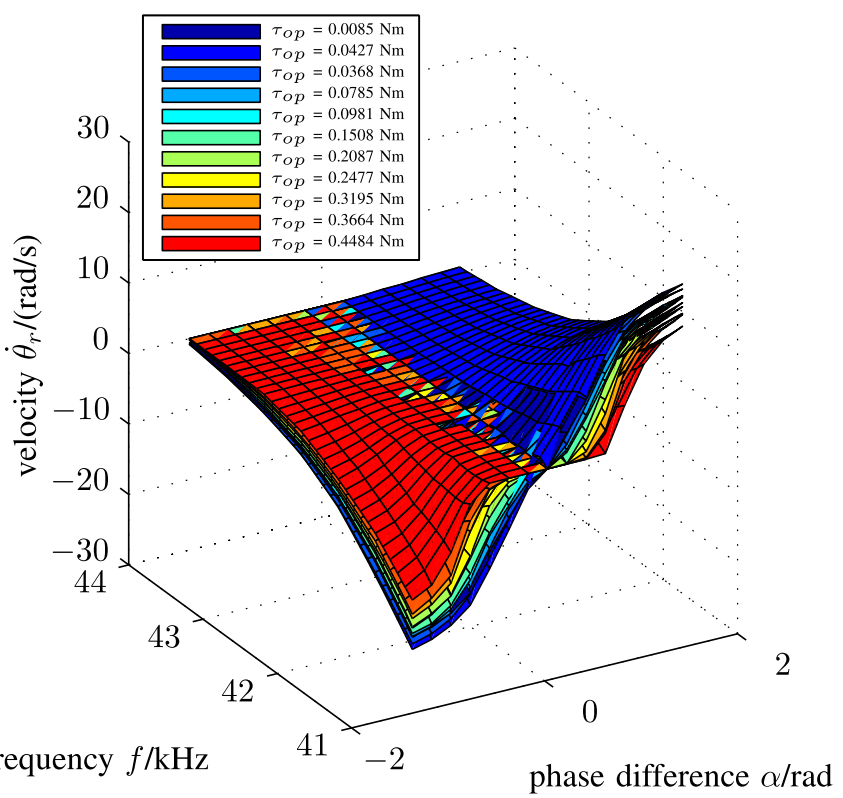

Fig. 8. Interpolated USM velocity, depending on phase difference, frequency and torque.

torque of $0.5 \mathrm{~N} \cdot \mathrm{m}$, since excessive torques may shorten the motor's lifetime. The velocity of the motor at a minimum opposing torque and for varying phase difference and frequency is depicted in Fig. 7. A complete interpolation of the data is depicted in Fig. 8.

\section{B. Methods for Model Parameter Identification}

In order to identify the USM model (10), (12), the brake simulates the opposing torque such that

$$
\tau_{o p}=\tau_{b} \operatorname{sgn}\left(\dot{\theta}_{r}\right)
$$


TABLE III

IDENTIFIEd MOdel Parameters AND FunCtions

\begin{tabular}{lc}
\hline \hline Parameter & Value \\
\hline$J_{r}$ & $17.2 \cdot 10^{-6} \mathrm{~kg} \cdot \mathrm{m}^{2}$ \\
$\tau_{m}$ & $0.5 \mathrm{~N} \cdot \mathrm{m}$ \\
$C_{r}$ & $2.46 \cdot 10^{-4} \mathrm{~N} \cdot \mathrm{m} \cdot \mathrm{s}$ \\
$z\left(\tau_{\mathrm{op}}\right)$ & $1+2 \tau_{\mathrm{op}}$ \\
$q_{0}$ & $1.233 \mathrm{rad} / \mathrm{N} \cdot \mathrm{m}$ \\
$q_{1}$ & $0.027 \mathrm{rad}$ \\
\hline \hline
\end{tabular}

where $\tau_{b}$ is the brake torque. Thus, the parameters $J_{r}, \tau_{m}, C_{r}$, and function $z\left(\tau_{\mathrm{op}}\right)$ remain to be identified.

First, the inertia $J_{r}$ is the sum of the inertia of the motor and the magnetic brake, which can be found in the datasheets. The torque producible by the motor is equivalent to the rated torque, which can also be found in the datasheet of the motor. A method for identifying the viscous friction coefficient $C_{r}$ of a similar model has been presented by García-Rochín et al. [15]. The same approach that is based on a step response and the time constant of the USM can be used here.

Finally, in order to quantify the impact of the load torque $\tau_{\text {op }}$ on the stator velocity $\omega_{\text {st }}$, the function describing the dead zone width is identified. It is described by $\alpha_{\mathrm{dz}}\left(\tau_{\mathrm{op}}\right)$ such that the motor stalls for all $|\alpha|<\left|\alpha_{\mathrm{dz}}\left(\tau_{\mathrm{op}}\right)\right|$. We assume a linear function as in [15] and define it piece-wise

$$
\alpha_{\mathrm{dz}}\left(\tau_{\mathrm{op}}\right)= \begin{cases}q_{0}+q_{1} \tau_{\mathrm{op}}, & \tau_{\mathrm{op}}>0 \\ {\left[-q_{0}, q_{0}\right],} & \tau_{\mathrm{op}}=0 \\ -q_{0}+q_{1} \tau_{\mathrm{op}}, & \tau_{\mathrm{op}}<0 .\end{cases}
$$

\section{Model Identification Results}

All parameters have been identified from datasheets and recorded data from the testbed. The inertia of the USR60 is $J_{m}=7.2 \cdot 10^{-6} \mathrm{~kg} \cdot \mathrm{m}^{2}$ and the magnetic brake has an inertia of $J_{b}=10^{-5} \mathrm{~kg} \cdot \mathrm{m}^{2}$. Therefore, the inertia of the test setup is $J_{r}=17.2 \cdot 10^{-6} \mathrm{~kg} \cdot \mathrm{m}^{2}$. The viscous friction can be identified as $C_{r}=2.46 \cdot 10^{-4} \mathrm{~N} \cdot \mathrm{m} \cdot \mathrm{s}$ [15]. Also using the datasheet of the $\mathrm{USM}$, the rated torque $\tau_{m}=0.5 \mathrm{~N} \cdot \mathrm{m}$ is identified. The relationship between the opposing torque $\tau_{\mathrm{op}}$ and the width of the dead zone $\alpha_{\mathrm{dz}}\left(\tau_{\mathrm{op}}\right)$ can be interpolated with the linear function such that $q_{0}=1.233 \mathrm{rad} / \mathrm{N} \cdot \mathrm{m}$ and $q_{1}=0.027 \mathrm{rad}$ [15]. Finally, the individual terms defining the stator velocity $\omega_{\text {st }}$ can be identified. The parameters of the exponential function are identified as $a=44, b=1$ by interpolation. The correction term is then interpolated as $z\left(\tau_{\mathrm{op}}\right)=1+2 \tau_{\mathrm{op}}$. The identified parameters of (10)-(12) are summarized as in Table III.

Plots of the dependency between phase difference, frequency, and velocity that were simulated with a time step of $1 \cdot 10^{-7} \mathrm{~s}$ are depicted in Fig. 9. A superposition of all curves at different opposing torques is depicted in Fig. 10. The model reproduces the sinusoidal shape of the phase difference dependency as well as the exponential shape of the frequency dependency. Dead zones are modeled, but hystereses are not captured. The root mean square (RMS) error of the model at different frequencies

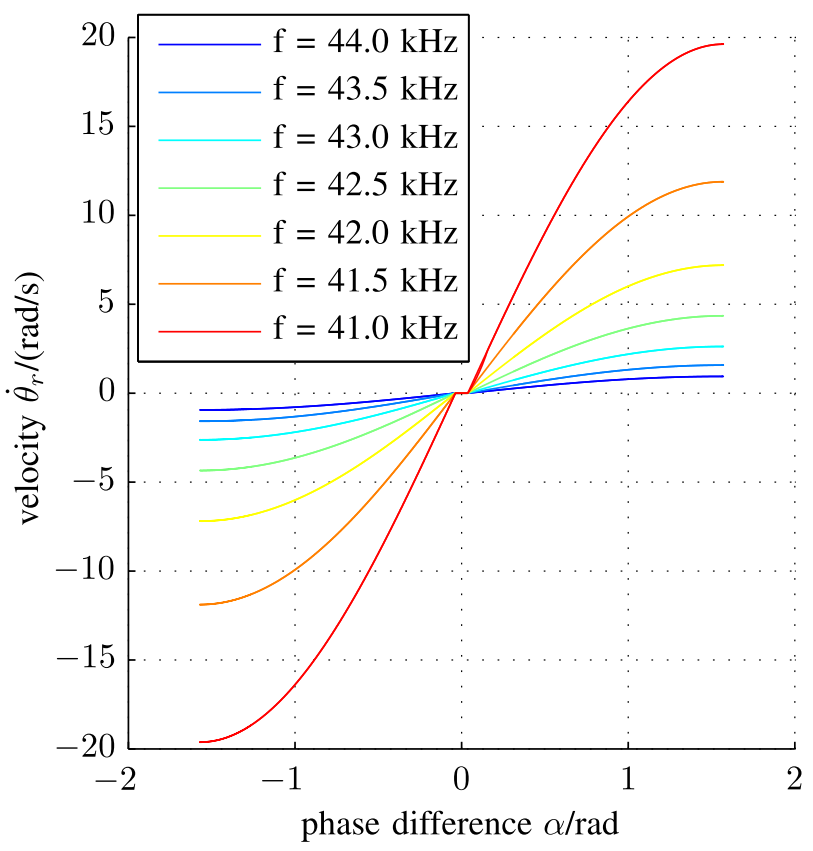

Fig. 9. Modeled velocity depending on phase difference and frequency at minimum load.

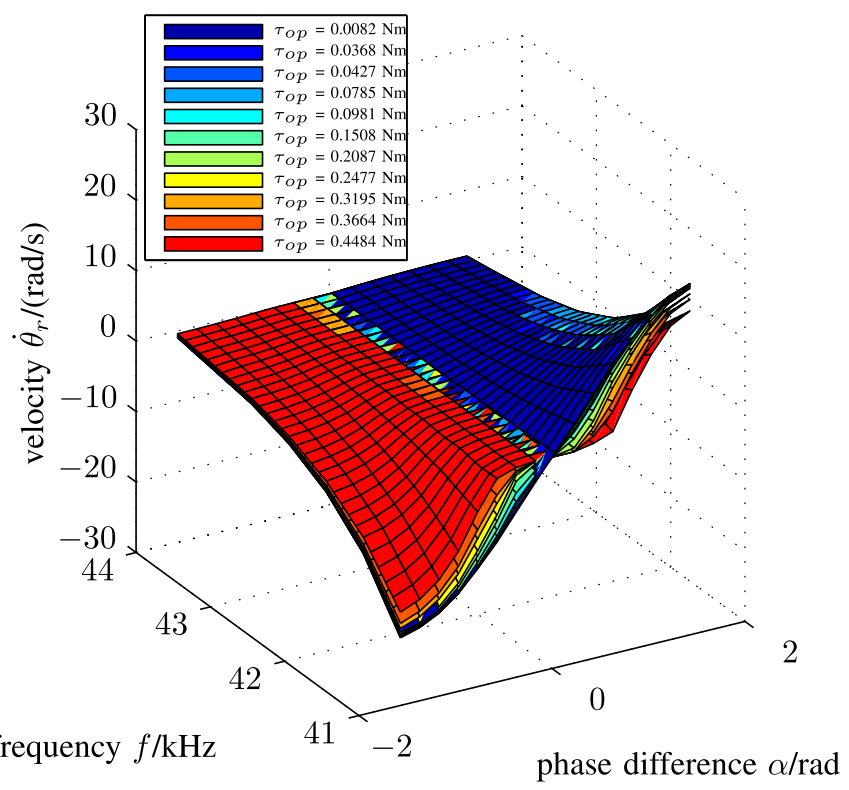

Fig. 10. Modeled USM velocity, depending on phase difference, frequency, and torque of the model.

and loads was computed: The maximum RMS error occurs for a minimum load at a minimum frequency and is $2.3 \mathrm{rad} / \mathrm{s}$. For higher loads it does not exceed $2.0 \mathrm{rad} / \mathrm{s}$ for any frequency. For frequencies higher than $42 \mathrm{kHz}$ the error does not exceed $1.2 \mathrm{rad} / \mathrm{s}$ for every load used during the identification.

\section{CONTROLler VALIDATION IN SimULATION AND EXPERIMENTS}

In order to validate both model and controller, the two-input controller was implemented on the testbed, using a sampling 


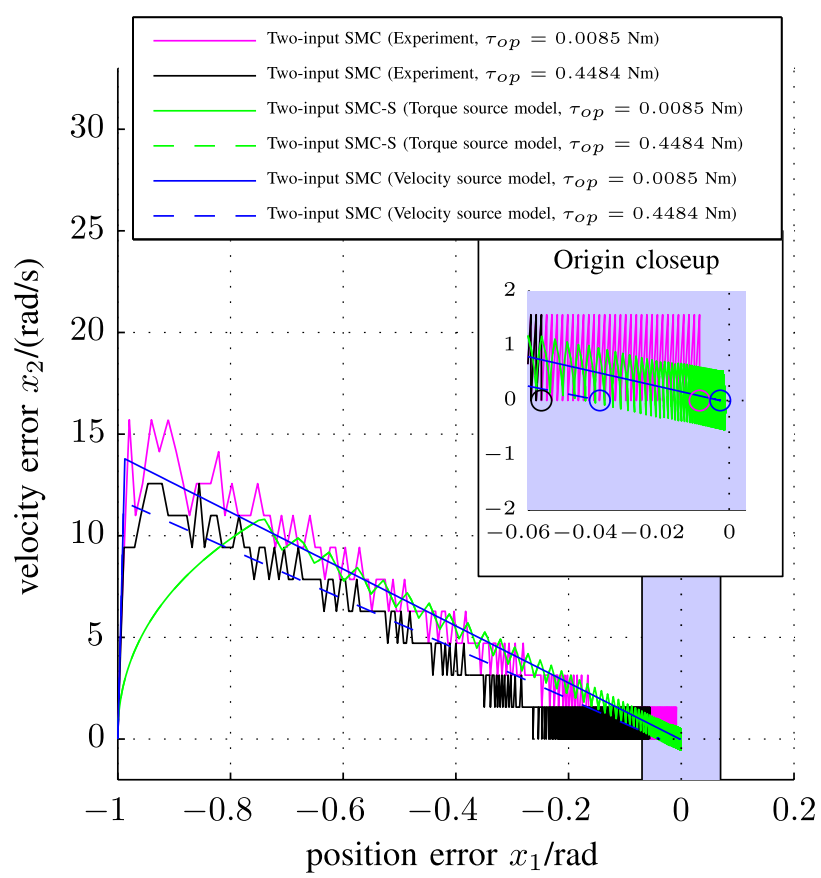

Fig. 11. Phase portrait of controlled systems in experiment and in simulation after a 1 rad position step input.

rate of $1 \mathrm{kHz}$, and simulated with the model, using a time step of $1 \cdot 10^{-8} \mathrm{~s}$. The reference trajectory $r_{\text {ref }}$ was a position step input of $1 \mathrm{rad}$ at opposing torques of $0.0085 \mathrm{~N} \cdot \mathrm{m}$ and $0.4484 \mathrm{~N} \cdot \mathrm{m}$. The controller gain was set to $m=\frac{C_{r}}{J_{r}}=14.30$. Note, that this a design choice and covers the case where the state dependency vanishes in (23). With the identified parameters of the model, listed in Table III, and using (27) the precision of the novel two-input controller can be estimated as $\left|\Delta x_{1}(0.0085 \mathrm{~N} \cdot \mathrm{m})\right| \leq$ $0.0026 \mathrm{rad}$ in the best case and $\left|\Delta x_{1}(0.4484 \mathrm{~N} \cdot \mathrm{m})\right| \leq$ $0.0383 \mathrm{rad}$ in the worst case. Higher controller gains $m$ can further decrease the remaining steady-state error as indicated in (27).

The corresponding phase portrait is depicted in Fig. 11, where the region of phase difference control using the model is highlighted by a shaded area. The width of this area is determined by parameter $a$, since the maximum frequency of the traveling wave determines the minimum velocity in the frequency domain. In order to show the performance of the velocity source model, the controller was also simulated with the torque source model by García-Rochín et al. [15]. Note that with the torque source model, a signum function has to be implemented in the controller to achieve a sliding mode.

The performance of the novel controller was further compared to other hybrid position controllers for USMs, using phase difference and frequency as control inputs: A dual SMC/Pcontroller with adaptive dead zone compensation [19] and a two-input $\mathrm{H} \infty$ controller [17].

Since the novel position controller is based on the work by Canudas-de-Wit [14], who developed a one-input velocity controller, it is also important to highlight the advancements. Canudas-de-Wit did not identify a USM to provide a control law $R\left(f, \omega_{n}\right)$ for the frequency domain [14] nor did he apply his controller to a real USM. Hence, it is difficult to compare our results in simulation or experiment. But to highlight the importance of the newly-introduced fine-grained phase difference control, our controller was also implemented as a one-input controller on the model.

The evaluation highlights, how the main issues of USMs are addressed by our novel controller. First of all and similar to standard SMCs [19], nonlinear dynamics are dealt with by reducing the order of the controlled system to a first-order one. Second, as in [17] and [19] low speeds are realized using the phase difference as a control input. This is in contrast to the work by Canudas-de-Wit [14], where low speeds are not realizable. Finally and unlike previous work [14], [17], [19], dead zones are reduced without creating chattering or overshoot effects. Moreover, the remaining steady-state error can be estimated.

\section{A. Comparison of Simulation and Experiment of the Novel Two-Input Controller}

For the novel two-input controller, implemented on the model and that abstracts the stator as a velocity source, the step response in the phase portrait in Fig. 11 shows a reaching and a sliding phase, with a slope of $m=14.30$ for a minimum opposing torque $\tau_{\text {op }}$. This is in accordance with the experimental results, where the same controller is used on the testbed. The position errors below a certain margin are only corrected by phase difference control, while the frequency is saturated. The frequency of oscillations increases close to the origin, as can be seen for the two-input SMC during the experimental validation, which should not be confused with chattering. This originates from the velocity quantization due to the resolution of the used encoder and sampling time. The model however, does not reproduce this effect, since it does not take into account the encoder precision. The remaining steadystate position error of the two-input SMC has a magnitude of $\left|\Delta x_{1, \exp }(0.0085 \mathrm{~N} \cdot \mathrm{m})\right|=0.0088 \mathrm{rad}$ in the experiment and is marked with a magenta circle in the closeup. The simulation has an error of $\left|\Delta x_{1 \text {, sim }}(0.0085 \mathrm{~N} \cdot \mathrm{m})\right|=0.0026 \mathrm{rad}$, as it has been estimated, and the endpoint is marked by a blue circle. Similar calculations can be done for the maximum load case. Note that the states come to a rest near the origin and do not oscillate infinitely. The discrepancy of the steady-state error results most likely from neglected friction effects and the interpolation of the model parameters. Moreover, the velocity source model overestimates the drop of maximum velocity under higher loads. Thus, the simulated slopes depicted in blue in Fig. 11 are not perfectly parallel.

Fig. 12 shows the velocity performance of the controlled system over time. For visibility, only the simulated system is depicted and the phase difference control domains are shown as shaded areas to the right. The velocity decreases in an exponential manner and converges to zero. Moreover, the smooth switch between the control domains is clearly visible. The time instant of the switch depends on the opposing torque and increases with increasing torque.

\section{B. Comparison of Velocity and Torque Source Model}

A comparison with a sliding mode based on a torque source model as in [15] can only be realized by adding an additional 


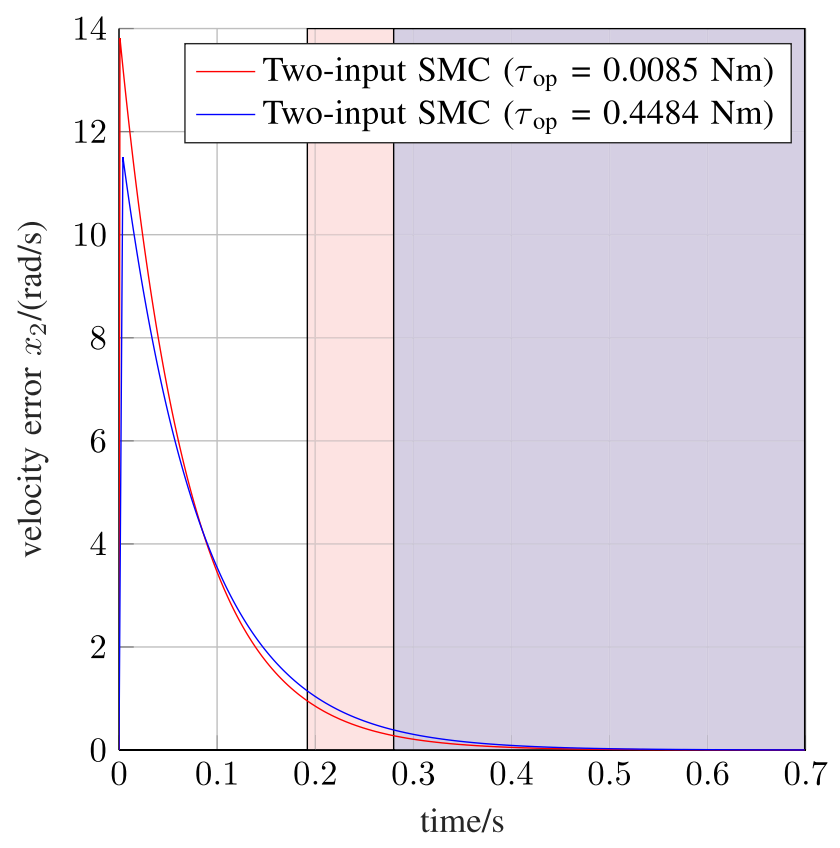

Fig. 12. Velocity error $x_{2}$ over time on the proposed velocity source model after a 1 rad position step input. The phase difference control domains in case of minimum and maximum opposing torque $\tau_{\mathrm{op}}$ start at different time instants. The domains are shown as the shaded orange and blue areas, respectively.

signum function to the two-input SMC. The simulation result of such a two-input SMC with signum function (SMC-S) with a torque source model is depicted in green in Fig. 11. In practice, the use of a signum function minimizes the steady-state error, but leads to chattering as can be seen in Fig. 11.

\section{Comparison With Other Two-Input Controllers}

The proposed velocity-source model is employed to compare the performance of the novel two-input controller to other hybrid position controllers for USMs using phase difference and frequency as control inputs. Senjyu et al. [19] introduced a dual $\mathrm{SMC} / \mathrm{P}$ controller, where the phase difference is controlled via an SMC controller and the frequency via a proportional controller. The reaction to a $1 \mathrm{rad}$ position step input is depicted in black in Fig. 13. The control switch takes place when $\alpha=\frac{\pi}{2} \mathrm{rad}$ and the frequency is controlled in the range of [41; 41.5] kHz. Hence, the control switch takes place at higher velocities, compared to the two-input SMC. This results in two disadvantages: First, the motor is mainly controlled in the phase difference control domain, which has serious consequences for the motor's already short lifetime [30]. Second, the control switch introduces a discontinuity of the velocity, which is highlighted with an arrow in Fig. 13. For haptic applications smoothness of the states is necessary. Finally, this controller foresees a digital implementation of a signum function for the SMC in the phase difference domain. This leads to chattering effects on a real USM and can be avoided, if the signum function is identified as a part of the USM.

Also, a two-input $\mathrm{H} \infty$ controller [17] has been proposed to control USMs via phase difference and frequency. However, in

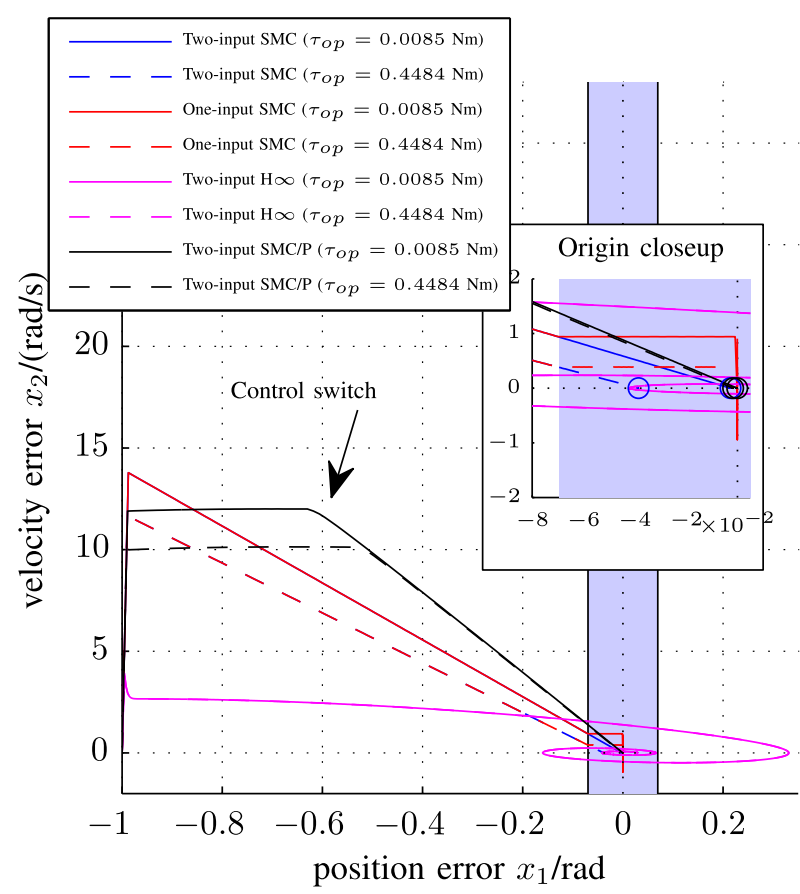

Fig. 13. Phase portrait of controllers on the proposed velocity source model after a 1 rad position step input.

TABLE IV

Time Until a Steady State Position With $x_{2}<0.01$ Rad $/ \mathrm{S}$ IS ReAched

\begin{tabular}{lcc}
\hline \hline Controller & $\tau_{\text {op }}=0.0085 \mathrm{~N} \cdot \mathrm{m}$ & $\tau_{\text {op }}=0.4484 \mathrm{~N} \cdot \mathrm{m}$ \\
\hline Two-input SMC & $0.52 \mathrm{~s}$ & $0.58 \mathrm{~s}$ \\
One-input SMC & $0.27 \mathrm{~s}$ & $0.46 \mathrm{~s}$ \\
Two-input $H_{\infty}$ & $>0.7 \mathrm{~s}$ & $>0.7 \mathrm{~s}$ \\
Two-input SMC/P & $0.39 \mathrm{~s}$ & $0.40 \mathrm{~s}$ \\
\hline \hline
\end{tabular}

the minimum load case it introduces a massive overshoot of the system, depicted in magenta in Fig. 13.

Finally, in order to compare our work to the original idea by Canudas-de-Wit [14], our controller has been implemented such that it only changes the sign of the phase difference. This is depicted in red in Fig. 13. Since the frequency saturates at a nonzero velocity, a position can only be held while oscillating around it with positive and negative velocities. Thus, chattering around zero remains for this controller.

The controllers are additionally compared using two performance indices: The time to reach a steady-state position and the control effort. The applied definition for a steady state is $x_{2}<0.01 \mathrm{rad} / \mathrm{s}$, whereas the control effort is expressed as the integral of $|\mu|$. Table IV shows the time required for the different controllers and load cases to reach a steady state. The one-input SMC performs best in the minimum load case, the two-input SMC/P controller performs best in the maximum load case, and the $H_{\infty}$ controller performs worst in both cases. The proposed two-input SMC reaches the steady state slower than the one-input SMC. The former reduces the velocity using phase differences in the whole range of $\left[-\frac{\pi}{2} ; \frac{\pi}{2}\right]$ rad and thus, is slower but avoids chattering around zero velocity. 
TABLE $V$

CONTROL EFFORTS EXPRESSED BY THE INTEGRAL OF $|\mu|$

\begin{tabular}{lcc}
\hline \hline Controller & $\tau_{\mathrm{op}}=0.0085 \mathrm{~N} \cdot \mathrm{m}$ & $\tau_{\mathrm{op}}=0.4484 \mathrm{~N} \cdot \mathrm{m}$ \\
\hline Two-input SMC & 1.06 & 1.54 \\
One-input SMC & 1.47 & 1.69 \\
Two-input $H_{\infty}$ & 3.01 & 3.01 \\
Two-input SMC/P & 8.53 & 8.53 \\
\hline \hline
\end{tabular}

Control efforts are used as a further comparison of the controllers. In order to compare the controllers on a common basis, $\mu$ is calculated for every controller type from the phase difference as well as frequency commands and using the control laws listed in Table I. The results of the integral of $|\mu|$ are listed in Table V. Clearly, the proposed two-input SMC performs best, whereas the two-input SMC/P controller performs worst for both cases. The control effort expresses some of the advantages that can be observed in Fig. 13: Chattering around zero velocity is avoided along with over-swinging, which add to an increased control effort.

\section{CONCLUSION}

We obtained a novel second-order model for rotary traveling wave USMs that abstracts the stator as a velocity source and includes a dynamic description of the dead zone. The dynamics of the stator was simplified and the model neglects the stator vibration velocity. We showed that a two-input sliding mode controller, using frequency and phase difference as commands, was GUAS for bounded disturbances in both control domains. This two-input hybrid position controller extends the velocity controller by Canudas-de-Wit to the position control case and allows fine-grained phase difference control. The novel controller performs better than a hybrid SMC/P or dual $\mathrm{H} \infty$ controller that was proposed in the literature, since it switches the control domains without a discontinuity of the velocity, reduces the amount of phase difference control and does not produce overshoot. In the future, we will employ the novel controller in an MR-compatible haptic interface together with an admittance control scheme.

\section{APPENDIX A USM MODEL BY CANUDAS-DE-WIT}

The USM model by Canudas-de-Wit [14] relies on the LuGre friction model. This friction model abstracts the friction interface as a bristle contact and includes stick-slip, Stribeck, Coulomb, viscous as well as other hysteretic characteristics, and is described by

$$
\begin{aligned}
\dot{z} & =\tilde{v}-\frac{|\tilde{v}|}{g(\hat{v})} z \\
F & =\sigma_{0} z+\sigma_{1} \dot{z}+\sigma_{2} \tilde{v} \\
g(\tilde{v}) & =F_{C}+\left(F_{S}-F_{C}\right) e^{\frac{-\tilde{v}^{2}}{v_{s}^{2}}} .
\end{aligned}
$$

Here, $\mathrm{z}$ is the bristle deflection, $\tilde{v}$ is the relative velocity, and $F$ is the tangential force at the friction interface. The parameters of the tangential force $F$ are the spring-like coefficient $\sigma_{0}=$ $1 / \epsilon$ as well as the microdamping and macrodamping friction coefficients $\sigma_{1}$ and $\sigma_{2}$, respectively. The nonlinear function $g(\tilde{v})$ includes the Coulomb friction force $F_{C}$ as well as the static friction force $F_{S}$ and depends on the Stribeck velocity $v_{s}$. In a singular-perturbed form, with the change of coordinates $\xi=$ $\sigma_{0} z=(1 / \epsilon) z,(36),(37)$ can be written as

$$
\begin{aligned}
\dot{\xi} & =\tilde{v}-\frac{|\tilde{v}|}{g(\hat{v})} \xi \\
F & =\zeta+\epsilon \sigma_{1} \dot{z} .
\end{aligned}
$$

The model states that a force $F$, which originates from a velocity difference $\tilde{v}$, is transmitted at the friction interface. In case of a USM, the velocity difference $\tilde{v}$ is the relative velocity of stator $\omega_{\text {st }}$ and rotor $\dot{\theta}_{r}$. Canudas-de-Wit obtained the stator velocity $\omega_{\text {st }}$ by modeling the traveling wave as superposition of two standing waves such that

$$
\omega_{\mathrm{st}}=V_{0} R\left(f, \omega_{n}\right) \operatorname{sgn}(\alpha) .
$$

Here, $V_{0}$ is the voltage applied to the piezoelectric material, $R(f)$ is a nonlinear function depending on the frequency of the traveling wave $f$ and $\alpha$ is the phase difference of the two standing waves.

Finally, by assuming that the rotor has an inertia $J_{r}$ as well as a damping coefficient $C_{r}$ and that it is driven by the torque that is transmitted at the friction interface

$$
J_{r} \ddot{\theta}_{r}+C_{r} \dot{\theta}_{r}=\tau_{d r}(\xi, \dot{\xi})
$$

the USM model by Canudas-de-Wit (1)-(4) is obtained.

\section{REFERENCES}

[1] C. Zhao, Ultrasonic Motors. Nanjing, China: Springer, 2011.

[2] Y. Liu, W. Chen, J. Liu, and X. Yang, "A high-power linear ultrasonic motor using bending vibration transducer," IEEE Trans. Ind. Electron., vol. 60 , no. 11 , pp. $5160-5166$, Nov. 2013.

[3] X. Zhou, W. Chen, and J. Liu, "Novel 2-DOF planar ultrasonic motor with characteristic of variable mode excitation," IEEE Trans. Ind. Electron. vol. 63, no. 11, pp. 6941-6948, Nov. 2016.

[4] F. Sergi, V. Chawda, and M. K. O'Malley, "Interaction control of a nonbackdriveable MR-compatible actuator through series elasticity," in Proc. ASME Dyn. Syst. Control Conf., 2013, pp. 21-23.

[5] M. A. Tavallaei, P. M. Johnson, J. Liu, and M. Drangova, "Design and evaluation of an MRI-compatible linear motion stage," Med. Phys., vol. 43, no. 1 , pp. 62-71, 2016

[6] M. Tavallaei, S. F. Atashzar, and M. Drangova, "Robust motion control of ultrasonic motors under temperature disturbance," IEEE Trans. Ind. Electron., vol. 63, no. 4, pp. 2360-2368, 2015.

[7] N. W. Hagood and A. J. McFarland, "Modeling of a piezoelectric rotary ultrasonic motor," IEEE Trans. Ultrason., Ferroelect. Freq. Control, vol. 42, no. 2, pp. 210-224, Mar. 1995

[8] M. T. El Hagry, A. A. Mahfouz, and H. S. Ahmed, "Experimental investigation of a mathematical model for traveling wave ultrasonic motors," in Proc. 4th Int. Conf. Appl. Math. Comput. Sci., 2005, pp. 1-10.

[9] T. Senjyu, S. Yokoda, and K. Uezato, "Position control of ultrasonic motors using sliding mode control with multiple control inputs," in Proc. 13th Annu. Appl. Power Electron. Conf. Expo., 1998, pp. 597-602.

[10] T. Senjyu, T. Kashiwagi, and K. Uezato, "Position control of ultrasonic motors using MRAC with dead-zone compensation," Trans. Ind. Electron., vol. 48 , no. 6, pp. $1278-1285$.

[11] T. Senjyu, M. Nakamura, N. Urasaki, H. Sekine, and T. Funabashi, "Mathematical model of ultrasonic motors for speed control," in Proc. Appl. Power Electron. Conf. Expo., 2006, pp. 290-295. 
[12] J. Zhang, T. Zhan, Z. Xie, and W. Wu, "Multivariable nonlinear model of ultrasonic motor based on Hammerstein Model and uniform design," in Proc. 8th World Congr. Intell. Control Autom., 2010, pp. 5794-5799.

[13] H. Yoshino and K. Tanaka, "Finite-time stabilization of ultrasonic motor with stochastic compensator for chattering phenomena," in Proc. 42nd Annu. Conf. Ind. Electron. Soc., 2016, pp. 160-165.

[14] C. Canudas de Wit, "Control design for ultrasonic motors with dynamic friction interface," in Proc. 14th World Congr. IFAC, 1999, pp. 970-974.

[15] R. García-Rochín, M. Kühne, R. Santiesteban-Cos, G. J. Rubio-Astorga, and A. Peer, "Second-order model for rotary traveling wave ultrasonic motors," in Proc. IEEE-RAS 15th Int. Conf. Humanoids Robots, 2015, pp. 991-996.

[16] T. Senjyu, H. Miyazato, and K. Uezato, "Quick and precise position control of an ultrasonic motor with dual mode control," Elect. Eng. Jpn., vol. 116, no. 3, pp. 1333-1340, 1996.

[17] T. Senjyu et al., "Position control of ultrasonic motors using two-control inputs HM controller," in Proc. Power Electron. Drive Syst., 2003, pp. 269-274.

[18] T. Senjyu, T. Yoshida, K. Uezato, and T. Funabashi, "Ultrasonic motors using adaptive backstepping control and dead-zone compensation with fuzzy inference," in Proc. IEEE Int. Conf. Ind. Technol., 2002, pp. 560-565.

[19] T. Senjyu, S. Yokoda, Y. Gushiken, and K. Uezato, "Position control of ultrasonic motors with adaptive dead-zone compensation," in Proc. IEEE Ind. Appl. Conf., 1998, pp. 506-512.

[20] T. Senjyu, H. Miyazato, S. Yokoda, and K. Uezato, "Position control of ultrasonic motors using neural network," in Proc. IEEE Int. Symp. Ind. Electron., 1996, pp. 1059-1066.

[21] C. Bartzis, K. Zarkadis, K. Zaoskoufis, A. Tzes, and E. C. Tatakis, "Experimental studies on a fuzzy-logic control scheme for an ultrasonic motor," in Proc. 2016 IEEE Conf. Control Appl., 2016, pp. 1123-1128.

[22] C. Canudas de Wit, H. Olsson, K. J. Aström, and P. Lischinsky, "A new model for control of systems with friction," IEEE Trans. Automat. Control, vol. 40, no. 3, pp. 419-425, Mar. 1995.

[23] Y. V. Orlov, Discontinuous Systems. Ensenada, Mexico: Springer, 2009.

[24] A. F. Filippov, Differential Equations with Discontinuous Right-Hand Side. Norwell, MA, USA: Kluwer, 1988.

[25] F. Giraud, B. Lemaire-Semail, J. Aragones, J. P. Robineau, and J. T. Audren, "Precise position control of a traveling-wave ultrasonic motor," IEEE Trans. Ind. Appl., vol. 43, no. 4, pp. 934-941, Jul./Aug. 2007.

[26] F. Giraud, P. Sandulescu, M. Amberg, B. Lemaire-Semail, and F. Ionescu, "Modeling and compensation of the internal friction torque of a traveling wave ultrasonic motor," IEEE Trans. Haptics, vol. 4, no. 4, pp. 327-331, Oct.-Dec., 2011.

[27] V. Utkin, Sliding Modes in Control Optimization. Moscow, Russia: Springer, 1992

[28] V. Utkin, J. Guldner, and J. Shi, Sliding Mode Control in ElectroMechanical Systems. Columbus, OH, USA: Taylor \& Francis, 2009.

[29] R. I. Leine and H. Nijmeijer, Dynamics and Bifurcations of Non-Smooth Mechanical Systems. Zurich, Switzerland: Springer, 2004.

[30] A. Ferreira and P. Minotti, "High-performance load-adaptive speed control for ultrasonic motors," Control Eng. Practice, vol. 6, no. 1, pp. 1-13, 1998.

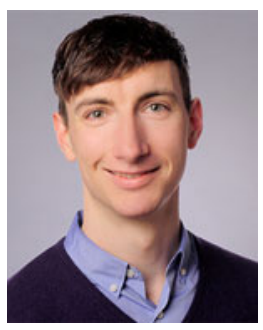

Markus Kühne received the Diploma Engineering degree in mechanical engineering from the Technical University of Munich, Munich, Germany, where he is currently working toward the Doctor of Engineering degree in electrical engineering. He also received the Diploma Engineering degree in robotics and embedded systems from the École Nationale Supérieure de Techniques Avancées ParisTech, Paris, France, and the Master's degree in robotics from the Université Pierre et Marie Curie, Paris, France.

His research interests include robot design and control as well as medical robotics.

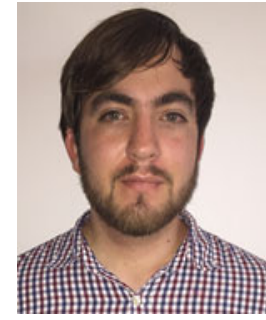

Roberto García Rochín (S'17) received the Bachelor's degree (Hons.) in mechatronic engineering with specialization in robotics and automation from the Tecnológico Nacional de México, Culiacán, Mexico, in 2015.

In 2016, he earned the Secretaria de EnergiaConsejo Nacional de Ciencia y Tecnologia scholarship to study the Master's degree in energy engineering from the Tecnológico de Monterrey, Monterrey, Mexico. In 2014, he received a scholarship from the DELFIN program and worked at the Technical University of Munich, Munich, Germany.

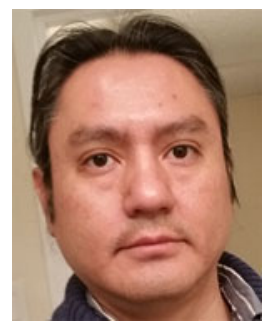

Raúl Santiesteban Cos received the Master's degree in applied mathematics engineering from the Instituto Potosino de Investigación Científica y Tecnologíca, San Luis Potosí, Mexico, in 2004. He received the $\mathrm{Ph} . \mathrm{D}$. degree in electronics and telecommunications with the option in control theory from the Center for Scientific Research and Higher Education at Ensenada, Ensenada, Mexico, in 2008

$\mathrm{He}$ is currently a Professor with the Tecnológico Nacional de México, Culiacán, Mexico. His research interests include control theory based on sliding modes with a focus on subactuated mechanical systems affected with dry friction and impulsive uncertainties.

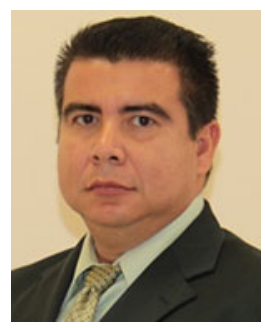

Guillermo Javier Rubio Astorga (M'10) was born in Culiacán, Mexico, in 1976. He received the Engineer's degree in electrical engineering from the Tecnológico Nacional de México, Instituto Tecnológico de Culiacán, Culiacn, Mexico, in 1998, the Masters degree in sciences in electrical engineering and the Doctor of Science degree in electrical engineering from the Advanced Studies and Research Center, National Polytechnic Institute, Guadalajara, Mexico, in 2001 and 2013 , respectively.

$\mathrm{He}$ is currently a Professor with the Department of Electronic-Electric Engineering, Tecnológico Nacional de México, Instituto Tecnológico de Culiacán, Culiacán, Mexico. His current research interests include the nonlinear control of electric machines.

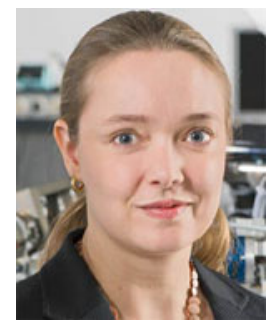

Angelika Peer (M'09) received the Diploma Engineering degree in electrical engineering and information technology and the Doctor of Engineering degree in electrical engineering from the Institute of Advanced Studies, Technical University of Munich, Munich, Germany in 2004 in 2008 , respectively.

She is currently a Full Professor with the Free University of Bozen-Bolzano, Bolzano, Italy. From 2014 to 2017, she was a Full Professor with the Bristol Robotics Laboratory, University of the West of England, Bristol, U.K. Before, she was a Senior Researcher and a Lecturer with the Institute of Automatic Control Engineering and TUM-IAS Junior Fellow of the Institute of Advanced Studies, Technical University of Munich, Munich, Germany. Her research interests include robotics, haptics, teleoperation, human-human and human-robot interaction, and human motor control. 